\title{
Fabrication of Glucose-Sensitive Layer-by-Layer Films for Potential Controlled Insulin Release Applications
}

\author{
TALUSAN Timothy Jemuel E., a , USMAN Ken Aldren S., b, \\ PAYAWAN Leon M. Jr. ${ }^{1, c}$ \\ 1 Institute of Chemistry, University of the Philippines, Diliman, Quezon City, Philippines \\ ${ }^{2}$ Natural Sciences Research Institute, University of the Philippines, Diliman, Quezon City, Philippines
}

\begin{abstract}
Self-regulated drug delivery systems (DDS) are potential alternative to the conventional method of introducing insulin to the body due to their controlled drug release mechanism. In this study, Layer-by-Layer technique was utlized to manufacture drug loaded, $\mathrm{pH}$ responsive thin films. Insulin was alternated with $\mathrm{pH}$-sensitive, [2-(dimethyl amino) ethyl aminoacrylate] (PDMAEMA) and topped of with polymer/glucose oxidase (GOD) layers. Similarly, films using a different polymer, namely Poly(Acrylic Acid) (PAA) were also fabricated. Exposure of the films to glucose solutions resulted to the production of gluconic acid causing a polymer conformation change due to protonation, thus releasing the embedded insulin. The insulin release was monitored by subjecting the dipping glucose solutions to Bradford Assay. Films exhibited a reversal in drug release profile in the presence of glucose as compared to without glucose. PAA films were also found out to release more insulin compared to that of the PDMAEMA films.The difference in the profile of the two films were due to different polymer-GOD interactions, since both films exhibited almost identical profiles when embedded with Poly(sodium 4-styrenesulfonate) (PSS) instead of GOD.
\end{abstract}

\section{Introduction}

Diabetes Mellitus, or hyperglycemia, is a chronic condition characterized by high glucose concentration in blood due to the insufficient amount of functional insulin [1]. The most common types of conventional insulin therapy to treat hyperglycemia make use of syringes, pens, and pumps. Insulin pens and syringes can cause tissue damage with repeated use and they provide immediate drug release in which the system releases the drug all at once. They also have the tendency to cause side-effects like hypoglycemia and anaphylaxis [2]. Insulin pumps provide controlled drug release by using glucose monitors. However, these pumps are expensive and are subject to computer error. Self-regulated drug delivery systems have a lot of advantages to that of conventional drug delivery systems. One of which is an easier control to the amount of released drug. As seen in figure 1, drugs have a minimum effective concentration (MEC) and a minimum toxic concentration (MTC).

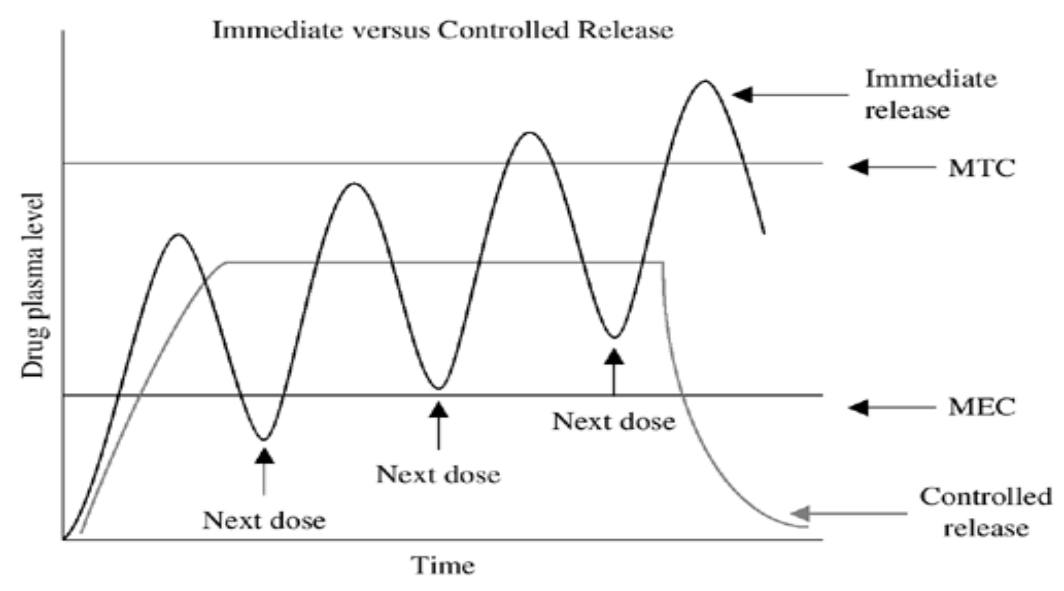

Figure 1. Controlled vs. immediate drug plasma release [3].

Corresponding author: ${ }^{a}$ tetalusan@up.edu.ph; ${ }^{b}$ kenaldrenusman@yahoo.com; ${ }^{\mathrm{c}} \underline{\text { Impayawan@yahoo.com }}$ 
Conventional drug delivery, has a tendency to reach the MTC when enough doses are reached because there is little control over the delivery rate of the drug. On the other hand, self-regulated drug delivery systems provide controlled drug release which maintains the amount of drug release at the optimum level $[3,4]$. Various studies for the use of $\mathrm{pH}$-responsive polymers have been studied and incorporated in films to achieve controlled insulin release. These films may be able to give a faster response due to their small sizes.

PDMAEMA and PAA are known pH-responsive polymers and are incorporated in a layer-by-layer film with insulin, the drug, and GOD. The drug release profile and on-off mechanism in response of glucose of the films were characterized using Atomic UV-Vis spectroscopy while film characterization was done using atomic force microscopy (AFM) and dynamic light scattering (DLS).

\section{Experimental}

\subsection{Fabrication of glucose-sensitive insulin- loaded films}

Films were fabricated using layer-by-layer deposition $[5,6]$. For drug release studies, deposition was done on glass substrates. For AFM characterization, quartz substrates were used. Aqueous solutions of PAA and PDMAEMA were prepared in aqueous $\mathrm{NaCl}$ as well as aqueous solutions of GOD. Insulin was dissolved in 100 $\mathrm{mL} \mathrm{HCl}$ and adjusted using $\mathrm{NaOH}$ to $\mathrm{pH}$ 6.0. Films with PSS embedded in the film instead of GOD were also prepared.

\subsection{Insulin release}

Characterization of the films with respect to the release of insulin was done using Bradford assay of the glucose solutions. Glucose was dissolved in PBS with $\mathrm{NaCl}$ for characterization. Drug release temperature was set to $37^{\circ}$ $\mathrm{C}$ to mimic body temperature. The films were immersed in the solutions of glucose in PBS buffer and an aliquot was extracted every 1 hour for a period of 10 hours. Aliquots were subjected to Bradford assay to determine amount of insulin released. A Coomasie Brilliant Blue solution was prepared by adding phosphoric acid to coomasie blue in $95 \%$ ethanol. After which distilled water was used to dilute the solution.

On-off release capability of film was also done by alternately dipping the film in $5 \mathrm{~g} / \mathrm{L}$ and $0 \mathrm{~g} / \mathrm{L}$ glucose solution. Switching was done every 1 hour for a period of 10 hours. Drug release conditions were the same as that of the drug release profile evaluation. Interaction of thin films with glucose solutions would make the system more acidic and introduce electrostatic repulsion in the polymer due to protonation.

\subsection{AFM imaging}

Deposition of layers was done in quartz and mica substrates for AFM imaging. Surface of the mica substrate was cleaved to expose a new layer ready for deposition of sample. The quartz substrate was cleaned and immersed in a piranha solution with 1:2 ratio of $\mathrm{H}_{2} \mathrm{O}_{2}$ and $\mathrm{H}_{2} \mathrm{SO}_{4}$ on a hotplate for 30 minutes. The quartz substrate was then washed with deionized water and dried. Analysis was done in the Condensed Matter Physics Laboratory (CMPL) NT-MDT SOLVER Scanning Probe Microscope at the National Institute of Physics, University of the Philippines-Diliman. A NSC05 $20^{\circ}$ cantilever was used in tapping mode in analysis. The samples were loaded unto a metal platform for the analysis of the topography of the sample. Analysis of data was done using a Nova computer software.

\subsection{Dynamic light scattering}

$\mathrm{pH}$ responsiveness of polymer was assessed using a Zeta Sizer Nano ZS90. $1 \mathrm{mg} / \mathrm{mL}$ aqueous solutions of PAA and PDMAEMA were prepared at various $\mathrm{pH}$ concentrations: $3,5,6,7,8$, and 10 .

\section{Results and discussion}

\section{$3.1 \mathrm{pH}$ responsiveness of the selected polymers}

Change in $\mathrm{pH}$ of solutions is caused by the production of gluconic acid from glucose catalyzed by GOD [7,8]. Figure 2 shows the dimensional response of the polymers to different $\mathrm{pH}$ environment. Both polymers exhibit an increase in their diameter at a low $\mathrm{pH}$ showing that both are $\mathrm{pH}$-sensitive. PAA increases its size drastically going to a more acidic $\mathrm{pH}$ compared to PMDAEMA.

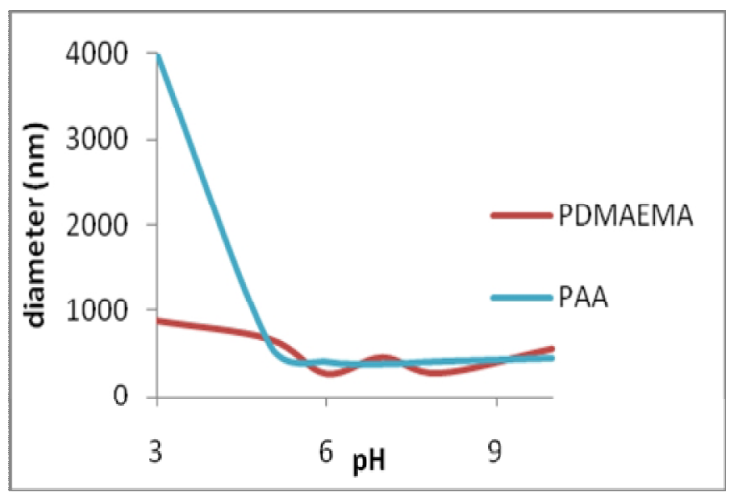

Figure 2. Dynamic light scattering profile of PDAMEMA and PAA.

For PAA, the removal of the hydrogen at a higher $\mathrm{pH}$ causes the molecule to become negatively charged, creating electrostatic repulsion between monomers. The aggregation of the acrylic acid monomers causes the significant increase in diameter of the PAA. Considering the average $\mathrm{Mv}$ of the PAA used which is $1.25 \mathrm{MDa}$, the aggregation of the molecules could cause the increase in diameter. Considering the structure PDMAEMA, the tertiary amine group PDMAEMA a weak electrolyte. Since PDMAEMA is a weak polyelectrolyte, the charge of the molecules depends highly on the $\mathrm{pH}$ of the 
polymer solution [5]. In contrast to PAA, PDMAEMA becomes charged at a lower $\mathrm{pH}$ environment. The repulsion between monomers causes the polymer to increase in size.

\subsection{Film fabrication}

Layer-by-layer deposition was done by dipping the glass substrate into alternating solutions of oppositely-charged electrolytes. The glass was activated using piranha solution resulting to a negative substrate surface charge. The positively-charged polymers were alternately deposited with the negatively-charged insulin and GOD via electrostatic interaction to form the film [9]. The mechanism and representational structure of the film can be seen in figure 3 .

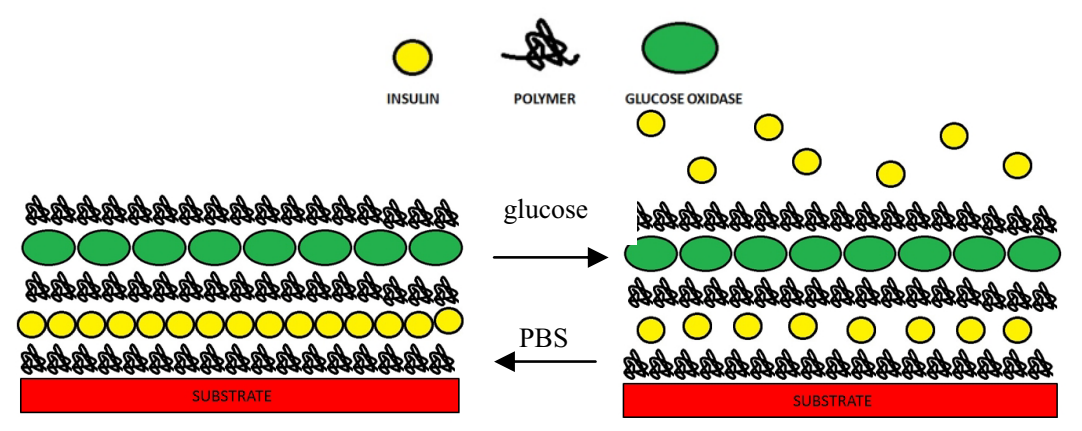

Figure 3. Film release mechanism.

\subsection{Film characterization}

AFM image of PAA and PDMAEMA films showed the topography of the as well as the average size and distribution of the particles in the film (figure $4 \& 5$ ).
Compared to the PAA films, the PDMAEMA films appeared to be larger and have a broader size distribution range. The PDMAEMA thin films also exhibit a rougher topography and there are pore-like structures on the surface which may contribute to its drug release.

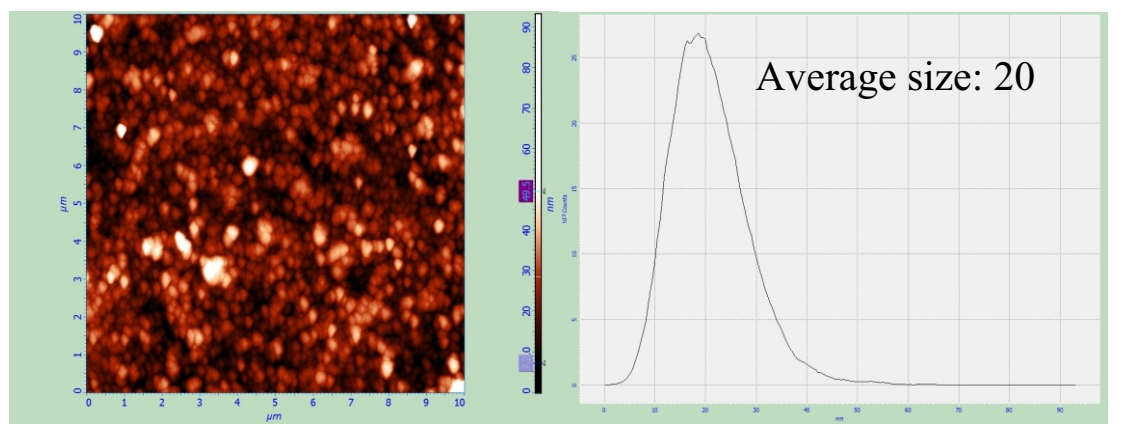

Figure 4. 10x10 $\mu \mathrm{m}^{2}$ PDMAEMA on quartz with histogram ( $10^{3}$ counts vs. particle size in $\left.\mathrm{nm}\right)$.

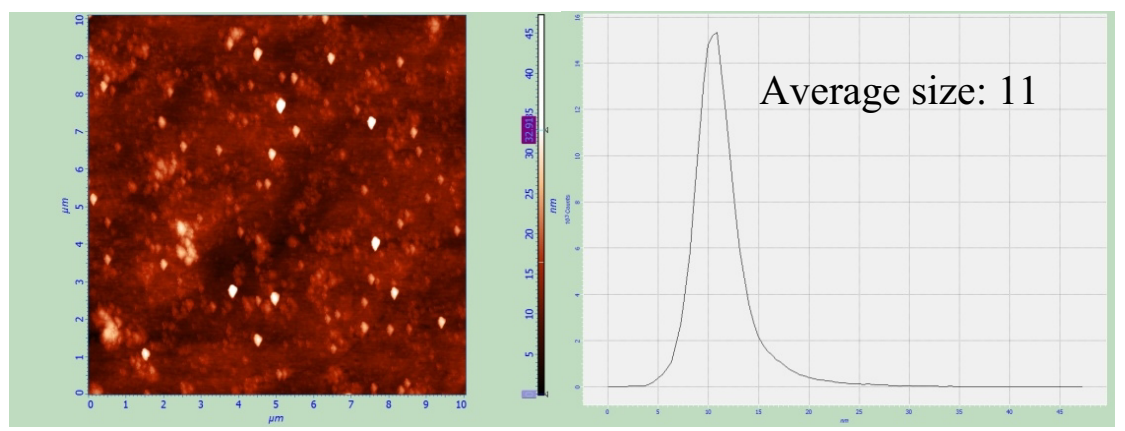

Figure 5. $10 \times 10 \mu \mathrm{m}^{2}$ PAA on quartz with histogram $\left(10^{3}\right.$ counts vs. particle size in $\left.\mathrm{nm}\right)$.

\subsection{Insulin release}

In vitro release of the drug from the PDMAEMA and PAA films was investigated using Bradford assay of the dipping solutions. Micro assay method using Bradford 
assay was done to quantify the amount of insulin released [10]. Drug release profiles of PAA and PDMAEMA show that the presence of glucose greatly affects how the insulin is released. As seen in figure 6, the release profile of the PDMAEMA thin film immersed in $0 \mathrm{~g} / \mathrm{L}$ glucose in PBS buffer is the inverse of the release profile of the PDMAEMA thin film immersed in $5 \mathrm{~g} / \mathrm{L}$ glucose solution. The same relationship between the two PAA films can be seen in figure 7. The PAA film immersed in a $0 \mathrm{~g} / \mathrm{L}$ glucose solution exhibit two maxima, one in the $3^{\text {rd }}$ hour and another in the $5^{\text {th }}$ hour. It also exhibits a minimum in the $4^{\text {th }}$ hour. For the PAA film immersed in a $5 \mathrm{~g} / \mathrm{L}$ glucose solution, there is a maximum ranging from the $3^{\text {rd }}$ to $4^{\text {th }}$ hour and two minima, each found in the $2^{\text {nd }}$ and $5^{\text {th }}$ hour. Reversal of release profile is also observed in PDMAEMA thin films. The PDMAEMA film immersed in $0 \mathrm{~g} / \mathrm{L}$ glucose solution show two minima, each found in the $3^{\text {rd }}$ and $5^{\text {th }}$ hour, and a maximum in the $4^{\text {th }}$ hour. For the PDMAEMA film immersed in $5 \mathrm{~g} / \mathrm{L}$ there are two maxima found in the $3^{\text {rd }}$ and $5^{\text {th }}$ hour and a minimum in the $4^{\text {th }}$ hour.

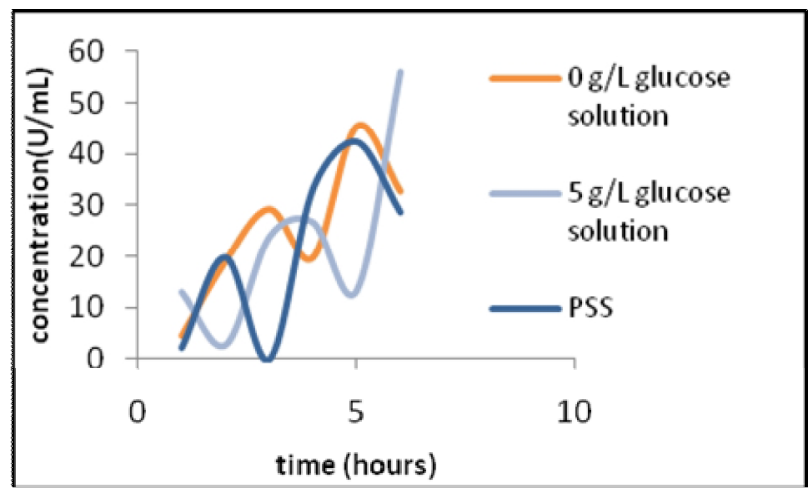

Figure 6. PDMAEMA film insulin release profile.

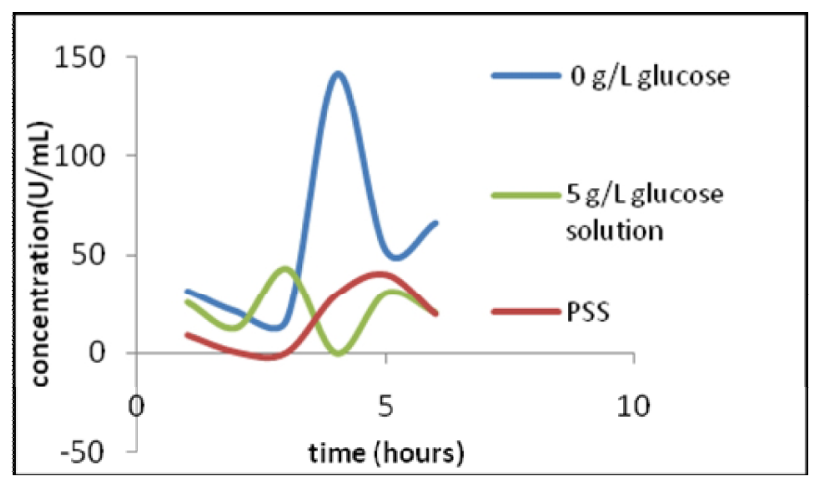

Figure 7. PAA film insulin release profile.

Looking at the release profiles of both polymers in 0 $\mathrm{g} / \mathrm{L}$ and $5 \mathrm{~g} / \mathrm{L}$ glucose solutions PAA and PDMAEMA drug release functionality are inversely proportional. Release profiles of the PAA-PSS thin film can be seen to be very similar and almost identical to that of the release profile of the PMDAEMA-PSS. However, the PSS thin films release profiles were very different from the release profiles of the films immersed in $0 \mathrm{~g} / \mathrm{L}$ glucose solutions. Polymer response may have been different because of the presence of GOD in the film.
On-off functionality of the thin films fabricated was also studied. As seen in figures $8 \& 9$, Both PAA and PDMAEMA thin films showed increase in release at the first 3 hours. Both also exhibited a sudden decrease in absorbance by the $4^{\text {th }}$ hour followed by a large release and again a decrease. On-off functionality of both films can be seen from the $2^{\text {nd }}$ hour up to the last hour. There is an increase in release when the film is immersed in a $5 \mathrm{~g} / \mathrm{L}$ glucose solution and a decrease when immersed in a $0 \mathrm{~g} / \mathrm{L}$ glucose solution. Amount release on the $1^{\text {st }}$ hour can be attributed to the film just starting to swell.

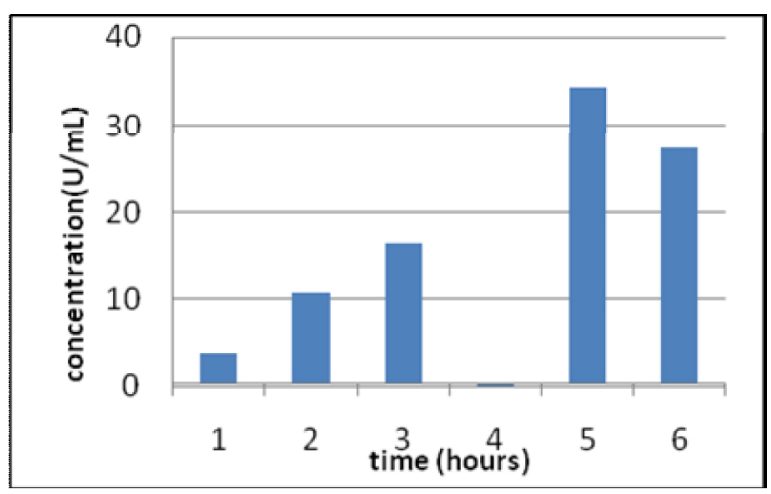

Figure 8. PAA on-off release.

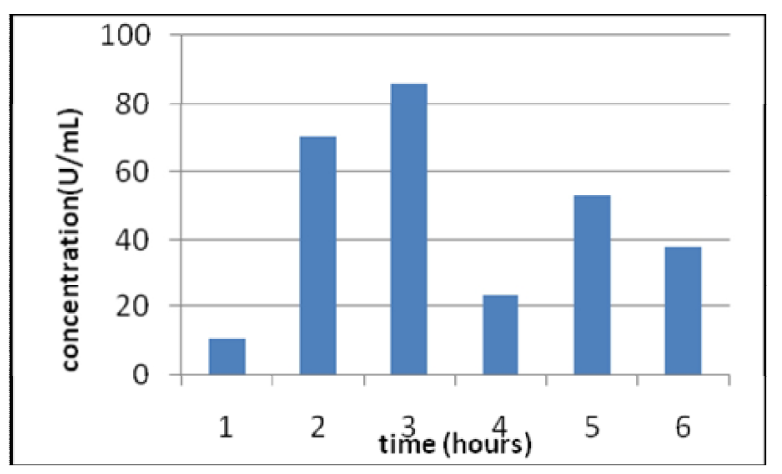

Figure 9. PDMAEMA on-off release.

\section{Conclusions}

Insulin was incorporated into a layer-by-layer film of $\mathrm{pH}$ sensitive, [2-(dimethyl amino) ethyl aminoacrylate] (PDMAEMA) and glucose oxidase (GOD). Likewise, another set of film was fabricated using Poly(Acrylic Acid) (PAA) for comparison. Exposure of the insulinPDMAEMA-GOD and films to high glucose concentrations results to the production of gluconic acid. Decreasing the $\mathrm{pH}$ caused changes in conformation of the polymer as a consequence, releasing the embedded insulin. The drug release was monitored by dipping the films into a glucose solution and subjecting the solutions to Bradford Assay. PDMAEMA films have significantly lower drug release compared to that of PAA films. PDMAEMA and PAA have different response in the presence of GOD. On contrary, PAA and PDMAEMA films embedded with PSS showed similar profiles.

\section{References}


1. E. Loghmani, Diabetes Mellitus: Type 1 and Type 2

2. S.R. Joshi, R.M. Parikh, A.K. Das, Supplement to Japi, 19-25 (2007)

3. M. Kutz, Standard Handbook of Biomedical Engineering and Design. n.d.

4. J. Siepmannn, R.A. Siegel, J. Rathbone, Springer, 19-43 (2012)

5. X. Chen, et al., Biomaterials 32, 1759-1766 (2011)
6. G. Decher, J.D. Hong, J. Schmitt, Thin Solid Films 210, 831-835 (1992)

7. E.O. Odebunmi, S.O. Owalude, J. Appl. Sci. Environ. Manage, 95-100 (2007)

8. J. Raba, H.A. Mottola, Critical Reviews in Analytical Chemistry 25, 1-42 (1995)

9. V. Ball, Materials 5, 2681-2794 (2012)

10. N.J. Kruger, The Protein Protocols Handbook, 2nd Edition. n.d., 15-21 